\title{
Coupled rotations in the lumbar spine - are these a consequence of passive spinal anatomy?
}

\author{
J. P. Little ${ }^{1,2}$, M. J. Pearcy ${ }^{2}$ \& C. J. Adam ${ }^{1,2}$ \\ ${ }^{1}$ Paediatric Spine Research Group, \\ Queensland University of Technology, Australia \\ ${ }_{2}^{2}$ Institute of Health and Biomedical Innovation, \\ Queensland University of Technology, Australia
}

\begin{abstract}
Previous studies have found that primary rotations in the lumbar spine are accompanied by coupled out-of-plane rotations, and abnormal coupled rotations have been associated with spinal pathology. However, it is not clear whether this accompanying rotation is due to passive (discs, ligaments and facet joints) or active (muscles) spinal anatomy. The aim of this study was to use a finite element model of the lumbar spine to determine three-dimensional rotations in the loaded spine, due to passive spinal anatomy alone. Computed tomography data for the bony anatomy of the visible man lumbar spine was used to generate a 3D osseoligamentous finite element model. Attachment origins and ligament stiffnesses for the major spinal ligaments were based on data reported in the literature. Loading conditions simulated physiologically observed rotations in the six degrees of rotational freedom, applied about in vivo centres of rotation. Model predictions were validated by comparison of intra-discal pressure and primary rotations with in vivo data and these showed good agreement. The computed coupled rotations for flexion, extension and axial rotation were similar to the in vivo results, with rotations within the first standard deviation of the reported in vivo data and in the same direction. Analysis of lateral bending consistently demonstrated higher coupled axial rotations than were observed in vivo, however, these were in the same direction as observed in vivo. The observed similarity in computed and in vivo nucleus pressures and primary rotations at each spinal level validated the models' ability to predict the in vivo response of the osseoligamentous lumbar spine. Coupled rotations due to primary motions in the sagittal and transverse planes appear to be caused by the passive spinal anatomy. However, for lateral bending, data for the computed coupled rotations suggest that the osseoligamentous anatomy alone does not account for the observed coupled rotation in vivo, and the muscles play a key role in the coupled response of the spine under this motion.

Keywords: finite element, spine, coupled motions, spinal loading.
\end{abstract}




\section{Introduction}

Previous clinical studies have found that primary rotations in the lumbar spine are accompanied by coupled out-of-plane rotations [1-5]. Pearcy [1] used biplanar radiography to measure in vivo three-dimensional rotations of the lumbar spine when healthy subjects actively rotated to their limits of lumbar motion. In a more recent study, Ochia et al. [2] measured three-dimensional lumbar rotations in vivo using computed tomography (CT) by applying external axial rotation while asking asymptomatic subjects to limit voluntary muscle action. Lund et al. [3] demonstrated a difference in coupled rotations between asymptomatic subjects and chronic low back pain patients. Coupled motions have also been investigated post-surgery, with both Lee et al. [4] and Natarajan et al. [5] finding a change in coupled rotations following facetectomy.

However, it is not clear from prior studies whether accompanying rotations are primarily due to passive (discs, ligaments, and facet joints) or active (muscles) spinal anatomy. Given the relationship between accompanying rotations and spinal pathology, it is important to investigate this question.

Accordingly, the aim of this study was to explore the role of passive spinal structures in generating coupled rotations in the normal human lumbar spine. To this end, a three dimensional finite element model of the osseoligamentous human lumbar spine was developed and subjected to loading conditions simulating physiological limits of rotational motion. Comparison of model predictions with in vivo data from healthy individuals [1] allowed the likely contribution of passive spinal structures to coupled motions to be assessed.

\section{Methods}

A three dimensional finite element (FE) model of a human osseoligamentous lumbar spine was developed using the Visible Man (VM) CT dataset (The Visible Human Project, U.S. National Library of Medicine).

\subsection{Geometry}

CT data for the VM lumbar spine were imported using custom Matlab software (The Mathworks, Natick, MA, USA) [6] and the dataset was thresholded to define the cortical bone. Using this software, user-selected landmarks on the bony anatomy of the spine (vertebrae, superior and inferior endplates, transverse processes, spinous processes, superior and inferior facet joint articulating surfaces) were defined and saved as three-dimensional coordinate points [6]. These data points were imported into a custom preprocessor (Python 2.4.3 programming language, Python Software Foundation) to generate threedimensional geometry for the osseous lumbar spine using parametric descriptions for each bony structure [7]. The external outline of each intervertebral disc was interpolated by sweeping between the profiles of the superior and inferior bounding endplates, and the internal annulus boundary was approximated by scaling the external outline about its centroid. 
The anterior longitudinal ligament, posterior longitudinal ligament, ligamentum flavum, inter-transverse ligaments, supra-spinous ligament and capsular ligaments were added to each intervertebral joint in the model. These were modelled between bony attachment sites and no wrapping of ligaments around adjacent bony structures was included in the model.

\subsection{Finite element mesh}

Using the custom preprocessor, the osseoligamentous model geometry was meshed for analysis using Abaqus 6.4.5 (Fig. 1). It was possible to control mesh density in each structure to optimize solution time and model accuracy. Table 1 gives details of the element types used for each spinal structure.

Table 1: $\quad$ Element types, material types and material parameters describing the spinal components. ( $\mathrm{E}=$ Young's modulus, $\mathrm{G}=$ Shear modulus, $v=$ Poisson's ratio, $\mathrm{C} 10$ and $\mathrm{C} 01=$ Mooney-Rivlin hyperelastic constants) [7-12].

\begin{tabular}{|l|l|l|l|l|}
\hline & Element type & Material type & $\begin{array}{l}\text { Material } \\
\text { Property }\end{array}$ & Reference \\
\hline Cancellous bone & $\begin{array}{l}\text { 3D-solid } \\
\text { continuum }\end{array}$ & Linear elastic & $\begin{array}{l}\mathrm{E}=140 \mathrm{MPa} \\
\mathrm{v}=0.2\end{array}$ & {$[9]$} \\
\hline Cortical bone & Shell & Linear elastic & $\begin{array}{l}\mathrm{E}=11300 \mathrm{MPa} \\
\mathrm{v}=0.2\end{array}$ & {$[9]$} \\
\hline Pedicles & Beam & Linear elastic & $\begin{array}{l}\mathrm{E}=200000 \\
\mathrm{G}=15000\end{array}$ & Quasi-rigid \\
\hline Facet joint - bone & Shell & Linear elastic & As for cortical & Quasi-rigid \\
\hline Transverse process & Beam & Linear elastic & $\begin{array}{l}\mathrm{E}=200000 \\
\mathrm{G}=150000\end{array}$ & Quasi-rigid \\
\hline Spinous process & Beam & Linear elastic & $\begin{array}{l}\mathrm{E}=200000 \\
\mathrm{G}=150000\end{array}$ & Quasi-rigid \\
\hline $\begin{array}{l}\text { Annulus ground } \\
\text { matrix }\end{array}$ & $\begin{array}{l}\text { 3D- solid } \\
\text { continuum }\end{array}$ & $\begin{array}{l}\text { Hyperelastic- } \\
\text { Mooney-Rivlin }\end{array}$ & $\begin{array}{l}\text { C10 }=0.7 \\
\text { C01 }=0.2\end{array}$ & {$[11]$} \\
\hline Collagen fibres & $\begin{array}{l}\text { Rebar - tension } \\
\text { only }\end{array}$ & $\begin{array}{l}\text { Linear elastic, } \\
\text { tension only }\end{array}$ & $\begin{array}{l}\mathrm{E}=500 \\
\mathrm{v}=0.3\end{array}$ & {$[10,12]$} \\
\hline Nucleus pulposus & 3D - hydrostatic & Hydrostatic fluid & Incompressible & {$[7,8]$} \\
\hline Ligaments & Connector & $\begin{array}{l}\text { Linear elastic, } \\
\text { tension only }\end{array}$ & $\begin{array}{l}\text { Refer Lu et al } \\
{[9]}\end{array}$ & {$[9]$} \\
\hline
\end{tabular}

Vertebral bodies were meshed using eight node hexahedral three-dimensional continuum elements for the internal cancellous bone, overlaid with four-node shell elements for the cortical shell. Quasi-rigid beam elements connected the posterior vertebra with the medial transverse processes (pedicles) and from the medial transverse processes to the medial spinous process (lamina). Beam elements were also used to represent the transverse and spinous processes, following the approach of previous studies $[13,14]$.

Three dimensional continuum elements simulated the annulus fibrosus ground matrix, with tension-only 'rebar' elements embedded at specific orientations to represent the annulus collagen fibres. These fibres were alternately orientated at $\pm 70^{\circ}$ in adjacent lamellae. Hydrostatic fluid elements were overlaid on the inner surface of the annulus to simulate a healthy, hydrostatic nucleus pulposus (Nachemson [8]). 
A simplified representation for the facet joints was used, whereby the bony surface of each articulating facet was simulated using shell elements and beam elements linked these faces to the lamina; simulating the superior and inferior articular processes. This representation for the articular processes and facet joints captured the detailed nonlinear geometry of the articulating facet surfaces, while reducing unnecessary mesh density in regions where localized analysis results were not imperative (the articular processes).

The interaction between adjacent facet joint faces was simulated using a finite-sliding frictionless tangential relationship [15] with 'softened' contact in the normal direction. The softened contact formulation simulated a nonlinear relationship between contact stress and separation distance, such that the contact pressure increases exponentially as the faces come into contact. This contact algorithm permitted only compressive forces to be transmitted across the joint and assumed an initial separation of $0.1 \mathrm{~mm}$ before the contact surfaces began to transmit force.

All ligaments were simulated as tension-only, axial connector elements (Table 1). Each capsular ligament was idealized as a single connector acting at the centre of the articulating joint in question.

A

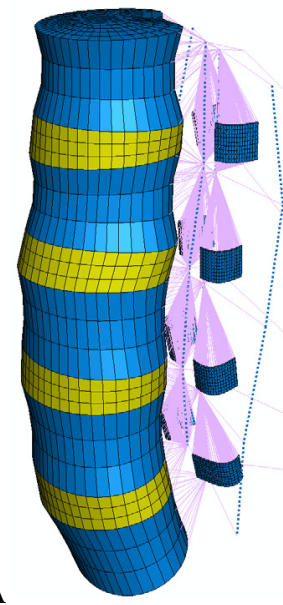

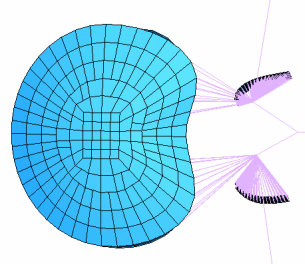

B

Figure 1: $\quad$ Finite element mesh A. Left anterior view of the full lumbar spine; B. Superior view of the L1 vertebra showing the posterior bony structures.

\subsection{Materials}

All osseous components were simulated using a linear elastic, isotropic constitutive behaviour (Table 1).

Higginson et al. [16] observed negligible fluid outflow from cartilage when tested at physiological loading rates. This finding was confirmed by Oloyede and Broom [17] and DiSilvestro et al. [18]. The current study aimed to simulate 
physiological loading rates, therefore, an assumption of incompressibility for the annulus ground substance was justified. Hyperelastic material laws effectively capture the nonlinear, incompressible response of the annulus ground substance $[19,20]$ and a Mooney-Rivlin material law was used [21]. Collagen fibre elements embedded in the annulus ground matrix were simulated using linear elastic, isotropic fibre constitutive behaviour. The nucleus pulposus was simulated as an incompressible, hydrostatic fluid [8].

Data for the ligament Young's modulii and cross-sectional areas were obtained from $\mathrm{Lu}$ et al. [9]. The lengths of connector elements defining the individual ligaments at each level of the spine were used to calculate individual ligament stiffnesses. To simulate the pre-stress resulting from the ligamentous connection between adjacent lumbar vertebra [22], the connector elements representing the supra-spinous ligament, inter-spinous ligament, inter-transverse ligament and ligamentum flavum were prestrained to $1 \%$. This ensured there was a slight initial lateral bulge in the annulus fibrosus of the discs.

\subsection{Loading and boundary conditions}

Physiological motions were simulated by applying a torso compressive force of $500 \mathrm{~N}$ (comparable to body weight above the lumbar spine [23]), as well as pure rotations simulating the limits of rotational motion defined by Pearcy [1]. The torso load was in a direction parallel to the spine axis and applied at a point in line with the centre of mass of the VM thoracolumbar spine, when viewed in the transverse plane. This point was rigidly constrained to the superior surface of the L1 vertebra.

Boundary conditions simulating primary physiological rotations were applied at the approximate centres of rotation (CR) for the lumbar spine. The CRs for sagittal plane rotations were measured from lateral radiographs of lumbar spines in the erect, fully extended and fully flexed postures [24]. The CRs for coronal and transverse plane rotations were derived from prior studies [25-27].

The CR for a particular rotation does not remain in the same location during the entire motion [28]. Therefore, the simulated CRs were free to move in translational degrees of freedom, which was equivalent to applying a pure moment. Hence, the primary and coupled rotations were not sensitive to changes in the initial CR location. The CR will tend to follow a locus with an expected endpoint calculated by comparing the rigid body motion between the vertebra at the beginning and end of the rotation. The initial locations of the CRs were thus determined using an iterative process, such that at the end of the simulated range of motion, the CR was at the expected (in vivo) endpoint.

Simulating the physiological motion of the spine as a pure rotation rather than by applying a bending moment ensured that the final configuration of the spine in the primary direction of rotation simulated the in vivo motion. The inferior surface of the L5 vertebra was constrained in all degrees of freedom during each analysis. The out-of-plane degrees of rotational freedom for the L1 vertebra were not constrained during the analyses, and the motion segments between L1 and L5 were constrained only by the simulated osseoligamentous anatomy. 


\subsection{Analysis}

The analyses were performed on an SGI Origin 3000 supercomputer (60 processors, 30GB RAM) using Abaqus 6.4.5. The analyses were implicit, quasistatic and incorporated nonlinear geometry.

Following the simulated torso compression load step, nucleus pulposus pressure and the amount of primary rotation in each motion segment were used to validate the model. Specifically, the ratio of nucleus pressure to applied pressure on the superior disc surfaces were calculated and compared to the value of 1.5 reported in vivo by Nachemson [8], and the primary rotations in each direction were compared with in vivo data from Pearcy [1] (Table 2).

Table 2: Physiological primary and coupled out-of-plane rotations in the lumbar spine from Pearcy [1]. Average in degrees (standard deviation).

\begin{tabular}{|c|c|c|c|c|}
\hline Motion & Joint & $\begin{array}{l}\text { Flexion (-ve) } \\
\text { /Extension } \\
\text { (+ve) rotation }\end{array}$ & $\begin{array}{l}\text { Lateral rotation } \\
\text { (Left +ve, } \\
\text { Right -ve) } \\
\end{array}$ & $\begin{array}{l}\text { Axial rotation } \\
\text { (Left +ve, } \\
\text { Right -ve) }\end{array}$ \\
\hline \multirow[t]{4}{*}{ Flexion } & L1-2 & $-8(6)$ & $1(1)$ & $1(1)$ \\
\hline & L2-3 & $-10(2)$ & $O(1)$ & $O(1)$ \\
\hline & L3-4 & $-12(1)$ & $1(1)$ & $-1(1)$ \\
\hline & L4-5 & $-13(4)$ & $\mathrm{O}(2)$ & $0(1)$ \\
\hline \multirow[t]{4}{*}{ Extension } & L1-2 & $5(2)$ & $0(1)$ & $1(1)$ \\
\hline & L2-3 & $3(2)$ & $0(1)$ & $0(1)$ \\
\hline & L3-4 & $1(1)$ & $O(1)$ & $O(1)$ \\
\hline & L4-5 & $2(1)$ & $-1(1)$ & $1(1)$ \\
\hline \multirow[t]{4}{*}{ Left lateral rotation } & L1-2 & $4(3)$ & $6(2)$ & $0(2)$ \\
\hline & L2-3 & $3(1)$ & $6(3)$ & $-1(1)$ \\
\hline & L3-4 & $2(2)$ & $5(3)$ & $-1(2)$ \\
\hline & L4-5 & $1(2)$ & $2(3)$ & $-1(2)$ \\
\hline \multirow[t]{4}{*}{ Right lateral rotation } & L1-2 & $2(2)$ & $-6(2)$ & $O(2)$ \\
\hline & L2-3 & $1(1)$ & $-5(1)$ & $1(1)$ \\
\hline & L3-4 & $1(2)$ & $-6(3)$ & $1(1)$ \\
\hline & L4-5 & $0(2)$ & $-3(2)$ & $1(1)$ \\
\hline \multirow[t]{4}{*}{ Left axial rotation } & L1-2 & $0(2)$ & $-3(2)$ & $1(1)$ \\
\hline & L2-3 & $0(1)$ & $-3(2)$ & $1(1)$ \\
\hline & L3-4 & $0(2)$ & $-3(2)$ & $2(1)$ \\
\hline & L4-5 & $0(3)$ & $-2(2)$ & $2(1)$ \\
\hline \multirow[t]{4}{*}{ Right axial rotation } & L1-2 & $0(2)$ & $3(2)$ & $-1(1)$ \\
\hline & L2-3 & $0(2)$ & $4(2)$ & $-1(1)$ \\
\hline & L3-4 & $0(1)$ & $3(2)$ & $-1(1)$ \\
\hline & L4-5 & $0(4)$ & $1(2)$ & $-1(1)$ \\
\hline
\end{tabular}

Predicted coupled rotations in the two out-of-plane directions were determined from the FE model results for each of the six primary motions (flexion, extension, left and right axial rotation, left and right lateral bending). The magnitude and direction of each primary and coupled rotation for each vertebra were compared with the data from Pearcy [1] (Table 2) and predicted coupled rotations that fell outside the first standard deviation from the experimental data were noted. 


\section{Results}

The results are presented in Table 3 .

\subsection{Model validation parameters}

The ratios between nucleus pulposus pressure and applied pressure in the four discs ranged from 1.42-1.59.

Primary rotations in the sagittal, coronal and transverse planes, calculated from the FE model, were generally either the same as the average in vivo rotations observed by Pearcy [1] or fell within the first standard deviation of the in vivo data. The three exceptions were for primary flexion and extension at the L3/4 disc $\left(10^{\circ}\right.$ and $3^{\circ}$, respectively), and primary left axial rotation at the L4/5 disc $\left(0^{\circ}\right)$. However, in each case these computed rotations fell within the range of data reported by Pearcy [1] - for flexion at L3/4, the range was $9^{\circ}$ to $14^{\circ}$ flexion; for extension at $\mathrm{L} 3 / 4$, the range was $4^{\circ}$ extension to $1^{\circ}$ flexion; for left axial rotation at $\mathrm{L} 4 / 5$, the range was $0^{\circ}$ to $3^{\circ}$ left rotation.

Table 3: $\quad$ Comparison of in vivo [1] and FE model data for coupled rotations in the lumbar levels; rotation (standard deviation) in degrees. (LB, lateral bending - positive LB = left rotation; SR, Sagittal rotation positive $\mathrm{SR}=$ extension; AR, Axial rotation - positive $\mathrm{AR}=$ left rotation). Shaded cells highlight motions for which the computed rotation fell outside the first standard deviation of the in vivo value. Columns denote applied primary rotations and rows denote the outof-plane coupled rotations per level.

\begin{tabular}{|c|c|c|c|c|c|c|c|c|c|c|c|c|}
\hline \multirow[t]{2}{*}{$\begin{array}{l}\text { Lumbar } \\
\text { Level }\end{array}$} & \multicolumn{2}{|c|}{ Flexion } & \multicolumn{2}{|c|}{ Extension } & \multicolumn{2}{|c|}{ Left Lateral Rotation } & \multicolumn{2}{|c|}{ Right Lateral Rotation } & \multicolumn{2}{|c|}{ Left Axial Rotation } & \multicolumn{2}{|c|}{ Right Axial Rotation } \\
\hline & In vivo & Computed & In who & Computed & in vio & Computed & In who & Computed & In wivo & Computed & In wo & Computed \\
\hline$\underset{\text { LB }}{\text { L1/2- }}$ & $1(1)$ & 1 & $O(1)$ & -1 & & & & & $-3(2)$ & 0 & $3(2)$ & 0 \\
\hline SR & & & & & $4(3)$ & 1 & $2(2)$ & 0 & $0(2)$ & 0 & $O(2)$ & 0 \\
\hline AR & $1(1)$ & -2 & $1(1)$ & 1 & $0(2)$ & -5 & $O(2)$ & 5 & & & & \\
\hline$\underset{\text { LB }}{\text { L2/3 - }}$ & $\alpha(1)$ & 1 & $0(1)$ & 0 & & & & & $-3(2)$ & -1 & $4(2)$ & 1 \\
\hline SR & & & & & $3(1)$ & 0 & $1(1)$ & 0 & $O(1)$ & 0 & $O(2)$ & 0 \\
\hline AR & $\alpha(1)$ & -2 & $0(1)$ & 0 & $-1(1)$ & -4 & $1(1)$ & 4 & & & & \\
\hline $\begin{array}{c}\text { L3/4 - } \\
\text { LB }\end{array}$ & $1(1)$ & 1 & $0(1)$ & 0 & & & & & $-3(2)$ & -1 & $3(2)$ & 1 \\
\hline SR & & & & & $2(2)$ & 0 & $1(2)$ & 0 & $0(2)$ & 0 & $0(1)$ & 0 \\
\hline AR & $-1(1)$ & -1 & $0(1)$ & 0 & $-1(2)$ & -5 & $1(1)$ & 4 & & & & \\
\hline $\begin{array}{l}\text { L4/5 - } \\
\text { LB }\end{array}$ & $\alpha(2)$ & 0 & $-1(1)$ & 0 & & & & & $-2(2)$ & -1 & $1(2)$ & 0 \\
\hline SR & & & & & $1(2)$ & 0 & $O(2)$ & 0 & $0(3)$ & 0 & $0(4)$ & 0 \\
\hline AR & $\alpha(1)$ & 0 & $1(1)$ & 0 & $-1(2)$ & -5 & $1(1)$ & 5 & & & & \\
\hline
\end{tabular}

\subsection{Out-of-plane coupled rotations}

Overall, the coupled motions for primary rotation in the sagittal plane (flexion/extension) were either the same as the average in vivo data [1] or within 
the first standard deviation (Table 3). An exception was for coupled axial rotation at L1/2 and L2/3 under primary flexion motion. These computed rotations were outside the reported in vivo range, however, the published dataset for the L1/2 level [1] only included six of the eleven individuals radiographed in the study. Similarly, for primary rotations in the transverse plane (left and right axial rotation), the computed coupled rotations were generally within the first standard deviation of the in vivo data. The only exceptions were for left axial rotation at the L1/2 level, where the accompanying lateral rotation was outside the range of reported in vivo data. In addition, for right axial rotation at the $\mathrm{L} 1 / 2$ and L2/3 levels the computed lateral rotations were below the first standard deviation of the in vivo data, but within the reported in vivo range. Primary rotations in the coronal plane (left and right lateral bending) resulted in computed coupled axial rotations considerably larger than the in vivo data. These coupled rotations generally fell outside both the standard deviation and reported range of in vivo measurements.

\section{Discussion}

The computational results presented in this study represent an attempt to explore the role of passive spinal structures in generating coupled out-of-plane rotations accompanying primary spinal motions. We presented a finite element model for a 'healthy' osseoligamentous lumbar spine with all ligaments intact and a hydrostatic nucleus pulposus because it is first necessary to understand the coupling mechanisms in the healthy spine before investigating pathological conditions.

Any new finite element model must be validated, and in this case the observed agreement between FE predictions and in vivo data for nucleus pressures and primary rotations at each spinal level demonstrated the ability of the model to correctly reproduce key aspects of the in vivo response of the lumbar spine. The geometric assumptions made in development of the model were intended to allow an anatomically detailed representation of the anterior column (vertebral bodies and intervertebral discs) while simplifying the posterior vertebral elements in line with the approach used by other modelling studies.

In this study, the loading applied to the model was formulated to simulate both the observed physiological ranges of motion and the centre of rotation about which this motion occurred. The applied rotations therefore produce the same net osseoligamentous motion as is produced by muscle activity in vivo, without explicitly modelling muscles or muscle forces. This approach is well suited for the current study, which aimed to identify the amount of coupled motion in the spine due to passive structures alone.

The key finding of this study is that for primary rotations in the sagittal or transverse planes, passive coupling inherent in the osseoligamentous spinal anatomy appears to account for in vivo levels of coupled out-of-plane rotation. For primary motion in the coronal plane however, the computed accompanying axial rotations exceeded in vivo values by up to $250 \%$ (although the computed axial rotations were in the same direction as the in vivo rotations). This suggests 
that for primary motions in the coronal plane, factors other than the osseoligamentous anatomy - presumably the active muscular structures of the lumbar spine - are of most importance in controlling the coupled axial rotation observed during primary lateral bending.

\section{References}

[1] Pearcy, M.J., Stereo radiography of lumbar spine motion. Acta Orthop Scand Suppl, 212, pp. 1-45, 1985.

[2] Ochia, R.S., Inoue, N., Renner, S., Lorenz, E., Lim, T.H., Andersson, G.B. \& An, H.S., Three-dimensional in vivo measurement of lumbar spine segmental motion. Spine, 31, pp. 2073-2078, 2006.

[3] Lund, T., Nydegger, T., Schlenzka, D. \& Oxland, T.R., Threedimensional motion patterns during active bending in patients with chronic low back pain. Spine, 27, pp. 1865-74, 2002.

[4] Lee, K.K., Teo, E.C., Qiu, T.X. \& Yang, K., Effect of facetectomy on lumbar spinal stability under sagittal plane loadings. Spine, 29, pp. 162431, 2004.

[5] Natarajan, R.N., Andersson, G.B., Patwardhan, A.G. \& Andriacchi, T.P., Study on effect of graded facetectomy on change in lumbar motion segment torsional flexibility using three-dimensional continuum contact representation for facet joints. J Biomech Eng, 121, pp. 215-21, 1999.

[6] De Visser, H., Evans, J.H., Little, J.P., Labrom, R., Askin, G., Pearcy, M.J. \& Adam, C.J., Sensitivity of patient-specific spinal finite element models to variation in bony anatomy derived from ct. 10th International Conference on Medical Image Computing and Computer Assisted Intervention, eds. Ayache, N. \& Ourselin, S., Springer, 2007.

[7] Little, J.P., Finite element analysis of anular lesions in the intervertebral disc [doctoral thesis] [Doctoral Thesis]. Queensland University of Technology, Brisbane, 2004.

[8] Nachemson, A., Lumbar intradiscal pressure: Experimental studies on post-mortem material. Acta Orthopaedica Scandinavica, 43, pp. 1960.

[9] Lu, Y.M., Hutton, W.C. \& Gharpuray, V.M., Do bending, twisting, and diurnal fluid changes in the disc affect the propensity to prolapse? A viscoelastic finite element model. Spine, 21, pp. 2570-9, 1996.

[10] Kumaresan, S., Yoganandan, N. \& Pintar, F.A., Finite element analysis of the cervical spine: A material property sensitivity study. Clin Biomech (Bristol, Avon), 14, pp. 41-53, 1999.

[11] Natali, A. \& Meroi, E., Nonlinear analysis of intervertebral disc under dynamic load. Transactions of the ASME Journal, 112, pp. 358-362, 1990.

[12] Ueno, K. \& Liu, Y.K., A three-dimensional nonlinear finite element model of lumbar intervertebral joint in torsion. $J$ Biomech Eng, 109, pp. 200-9, 1987.

[13] Andriacchi, T., Schultz, A., Belytschko, T. \& Galante, J., A model for studies of mechanical interactions between the human spine and rib cage. J Biomech, 7, pp. 497-507, 1974. 
[14] Lafage, V., Dubousset, J., Lavaste, F. \& Skalli, W., 3d finite element simulation of cotrel-dubousset correction. Comput Aided Surg, 9, pp. 1725, 2004.

[15] Kimpara, H., Lee, J.B., Yang, K.H., King, A.I., Iwamoto, M., Watanabe, I. \& Miki, K., Development of a three-dimensional finite element chest model for the 5th percentile female. Stapp Car Crash Journal, 49, pp. 251-269, 2005.

[16] Higginson, G.R., Litchfield, M.R. \& Snaith, J., Load-displacement characteristics of articular cartilage. International Journal of Mechanical Sciences, 18, pp. 481-86, 1976.

[17] Oloyede, A. \& Broom, N.D., A physical model for the time-dependent deformation of articular cartilage. Connective Tissue Research, 29, pp. 251-261, 1993.

[18] DiSilvestro, M.R., Zhu, Q. \& Suh, J.-K.F., Biphasic poroviscoelastic simulation of the unconfined compression of articular cartilage: Ii effect of variable strain rates (technical brief). Journal of Biomechanical Engineering, 123, pp. 198-200, 2001.

[19] Natali, A.N., A hyperelastic and almost incompressible material model as an approach to intervertebral disc analysis. J Biomed Eng, 13, pp. 163-8, 1991.

[20] Rohlmann, A., Zander, T., Schmidt, H., Wilke, H.-J. \& Bergmann, G., Analysis of the influence of disc degeneration on the mechanical behaviour of a lumbar motion segment using the finite element method. Journal of Biomechanics, 39, pp. 2484-2490, 2006.

[21] Schmidt, H., Heuer, F., Simon, U., Kettler, A., Rohlmann, A., Claes, L. \& Wilke, H.-J., Application of a new calibration method for a threedimensional finite element model of a human lumbar annulus fibrosus. Clinical Biomechanics, 21, pp. 337-344, 2006.

[22] Nachemson, A.L., Evans, J.H., Some mechanical properties of the third human lumbar interlaminar ligament (ligamentum flavum). J Biomech, 1, pp. 211-220, 1968.

[23] Nachemson, A.L., Disc pressure measurements. Spine, 6, pp. 93-7, 1981.

[24] Pearcy, M.J. \& Bogduk, N., Instantaneous axes of rotation of the lumbar intervertebral joints. Spine, 13, pp. 1033-41, 1988.

[25] Rolander, S.D., Motion of the lumbar spine with special reference to the stabilizing effect of posterior fusion. Acta Orthopaedica Scandinavica, 90, pp. 1-144, 1966.

[26] Cossette, J.W., Farfan, H.F., Robertson, G.H. \& Wells, R.V., The instantaneous centre of rotation of the third lumbar intervertebral joint. Journal of Biomechanics, 4, pp. 149-153, 1971.

[27] Adams, M.A. \& Hutton, W.C., The relevance of torsion to the mechanical derangement of the lumbar spine. Spine, 6, pp. 241-8, 1981.

[28] Seligman, J.V., Gertzbein, S.D., Tile, M. \& Kapasouri, A., Computer analysis of spinal segment motion in degenerative disc disease with and without axial loading. Spine, 9, pp. 566-73, 1984. 